\title{
Os efeitos do alfabetismo funcional sobre a empregabilidade dos trabalhadores brasileiros
}

\author{
Cristiano Machado Costa* \\ José Guilherme Cardoso Correa**
}

\begin{abstract}
Este trabalho utiliza a pesquisa Indicador de Alfabetismo Funcional (Inaf), realizada entre 2001 e 2007, para investigar os efeitos do alfabetismo funcional sobre a empregabilidade dos trabalhadores brasileiros, ressaltando as diferenças entre sexos e setores econômicos. Utilizando modelos probit e controlando para características observáveis dos indivíduos, os resultados apontam para uma maior importância das habilidades funcionais entre as mulheres. O efeito do aumento em um desvio-padrão na nota do Inaf resulta em crescimento de 6,10 p.p. na probabilidade de a mulher estar empregada. 0 indicador não se mostra significativo com relação à empregabilidade dos homens brasileiros, exceto no setor comercial, em que o aumento de um desvio-padrão no score Inaf eleva a probabilidade de um indivíduo estar empregado em 4 p.p. O setor comercial caracteriza-se pela maior interação entre os profissionais e os clientes, demandando habilidades funcionais que, muitas vezes, não são capturadas por meio das estatísticas usuais de escolaridade (nível e/ ou anos de estudo).
\end{abstract}

Palavras-chave: Empregabilidade. Alfabetismo. Capital humano.

\footnotetext{
*Programa de Pós-Graduação em Ciências Contábeis da Universidade do Vale do Rio dos Sinos - Unisinos, São Leopoldo-RS, Brasil (cmcosta30@gmail.com).

** Fundação Instituto Capixaba de Pesquisas em Contabilidade, Economia e Finanças, Vitória-ES, Brasil (zeguijo@gmail.com).
} 


\section{Introdução}

De acordo com a pesquisa Indicador de Alfabetismo Funcional (Inaf), em 2007 a taxa de analfabetismo (quando a pessoa não sabe ler e escrever) correspondia a 7,64\% no Brasil. ${ }^{1}$ Esse indicador, porém, não é homogêneo entre as regiões brasileiras. O Nordeste possui os Estados com as maiores taxas de analfabetismo no país, sendo que em todos os Estados dessa região os valores encontram-se acima da média nacional. No Piauí, Ceará, Pernambuco e Alagoas, praticamente, um em cada cinco habitantes era analfabeto, segundo a pesquisa.

As taxas de analfabetismo funcional - quando a pessoa é considerada incapaz de utilizar a leitura, a escrita e suas habilidades matemáticas para fazer frente às demandas de seu contexto social - são ainda maiores (INSTITUTO PAULO MONTENEGRO, 2006). A mesma pesquisa reporta uma taxa de $34,27 \%$ de pessoas que eram analfabetas funcionais ou possuíam um nível de habilidade funcional considerado rudimentar.

O analfabetismo funcional é uma medida de como os conhecimentos básicos aprendidos nos primeiros anos de escolaridade são transformados em habilidades necessárias para a realização de atividades cotidianas, como ler as instruções de uso de um medicamento, entender as leis de trânsito, ou ainda saber calcular os juros de um pagamento parcelado. $\mathrm{O}$ analfabeto funcional sabe ler e escrever, mas possui limitações em certas habilidades requeridas em tarefas remuneradas. A simples atividade de ler, compreender e executar, corretamente, as instruções delegadas por um superior requer o uso de capacidades funcionais. Essas habilidades são fundamentais para a empregabilidade dos indivíduos, principalmente para aqueles que possuem baixos níveis de escolaridade (medido em anos de estudo ou em número de séries completadas).

Este trabalho utiliza o Indicador de Alfabetismo Funcional (Inaf) para investigar a relação entre analfabetismo funcional e empregabilidade dos trabalhadores brasileiros. O Inaf é resultado de uma pesquisa conduzida pelo Ibope, em apoio à ação social realizada pelo Instituto Paulo Montenegro, e foi feita de 2001 a 2005 e novamente em 2007, quando passou a ser bianual.

De acordo com a metodologia da pesquisa (INSTITUTO PAULO MONTENEGRO, 2007), o alfabetismo funcional é dividido em quatro níveis: analfabeto, rudimentar, básico e pleno. 0 indicador mensura os níveis de alfabetismo funcional da população brasileira entre 15 e 64 anos de idade, englobando residentes em zonas urbanas e rurais de todas as regiões do Brasil. Em entrevistas domiciliares, foram aplicados questionários e testes práticos que mensuram a capacidade de leitura e os cálculos matemáticos básicos, sempre aplicados a um contexto social. Em média, 37,28\% dos homens são classificados como analfabetos ou em nível rudimentar, enquanto as mulheres pertencentes a esse grupo correspondem a $36,97 \%$ do total.

Com o objetivo de entender como o nível de alfabetismo funcional afeta a probabilidade de uma pessoa estar ou não empregada, foi elaborado um modelo econométrico do tipo

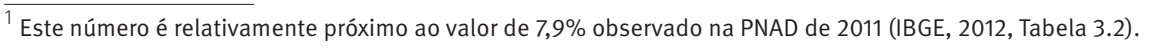


probit, no qual a variável dependente é igual a um, se o indivíduo está empregado, e igual a zero, caso ele esteja desempregado. As variáveis independentes buscam medir os efeitos das características individuais (sexo, cor e idade), do nível de escolaridade do indivíduo e dos pais e da medida de alfabetismo funcional, calculado pelo Inaf. Essa abordagem é importante para diferenciar o efeito puro do aumento da escolaridade sobre a empregabilidade, dissociando as medidas tradicionais de habilidades cognitivas das habilidades funcionais. Foram incluídas também variáveis que buscam mensurar as características regionais e conjunturais de cada mercado de trabalho, fundamentais para a determinação do emprego. As variáveis incluem o ano da pesquisa, o Estado de residência do entrevistado e as características da cidade. 0 objetivo é medir o efeito do Inaf, uma vez controladas as demais características da pessoa e do ambiente em que ela se encontra.

No sentido de evidenciar diferenças entre os mercados de trabalho para homens e mulheres, o modelo foi estimado em três especificações distintas, permitindo que o efeito do Inaf fosse diferente entre os sexos. Atentar para esta possibilidade é importante, pois muitas vezes homens e mulheres possuem escolhas diferentes em termos de setores e funções ao entrarem no mercado de trabalho. Além disso, foram testados dois modelos diferentes para cada especificação: um utilizando o score bruto (em números), obtido pelo indivíduo; e outro empregando variáveis binárias para cada uma das categorias (analfabeto, rudimentar, básico e pleno).

Os resultados das estimativas mostram que o score Inaf tem impacto positivo sobre a empregabilidade, mesmo após ter sido controlado para diversas características individuais. Em média, o efeito marginal do aumento de um desvio-padrão (aproximadamente 30 pontos) no score Inaf eleva em 2 pontos percentuais a probabilidade de se estar empregado. Os resultados também indicam que o impacto de uma melhor pontuação obtida no score Inaf é maior (e significativo) entre as mulheres. O efeito marginal do aumento de um desvio-padrão no score Inaf eleva em aproximadamente 6 p.p. a probabilidade de a mulher estar empregada. Este resultado pode indicar que as mulheres com maiores niveis de habilidades funcionais possuem mais chances de estarem empregadas, possivelmente em virtude dos segmentos de trabalho em que atuam, os quais costumam requerer, por exemplo, maior interação entre os profissionais e os clientes (serviços).

A teoria econômica e a evidência empírica ensinam que o aumento da escolaridade eleva a probabilidade de o trabalhador estar empregado. Entretanto, para baixos níveis de escolaridade, a capacidade de o indivíduo transformar sua escolaridade (medida em anos de estudo) em produtividade depende da funcionalidade do seu conhecimento. É possível, por exemplo, que dois indivíduos que possuam apenas a quarta série primária tenham diferentes níveis de alfabetismo funcional.

Para investigar esta possibilidade, reduziu-se a amostra para apenas aqueles indivíduos (homens e mulheres) com escolaridade até o ensino fundamental completo. ${ }^{2} \mathrm{O}$ objetivo foi

\footnotetext{
${ }^{2}$ A pesquisa Inaf utiliza a terminologia "primário" para as quatro primeiras séries do ensino fundamental, "ginásio" para as quatro últimas séries deste ensino e "colegial” para o ensino médio.
} 
verificar se o efeito marginal do alfabetismo funcional sobre a empregabilidade era maior entre os menores níveis de escolaridade. Os resultados mostram que, uma vez controladas as características individuais e regionais, o aumento de um desvio-padrão no score Inaf eleva em aproximadamente 4 p.p. a probabilidade de uma mulher que possui nível de escolaridade menor ou igual ao ensino fundamental completo estar empregada. Esse resultado é de magnitude similar, porém um pouco menor, ao encontrado para a amostra com todos os níveis de escolaridade. Portanto, os efeitos das habilidades funcionais não parecem ser maiores entre aqueles de escolaridade inferior, como se imaginava inicialmente.

Por fim, buscando entender mais detalhadamente a relação entre atividades e o efeito do alfabetismo funcional sobre as mulheres, foram testadas novamente as especificações iniciais, mas em três amostras distintas: uma com apenas indivíduos que trabalham no setor de prestação de serviços; outra com aqueles alocados na indústria de transformação; e a terceira com os indivíduos do setor comercial. A ideia é verificar se existem requerimentos funcionais que são mais demandados em um setor do que em outro.

Enquanto os resultados não são significantes estatisticamente nos setores de prestação de serviços (em que se encontram muitos profissionais liberais) e na indústria (cujo trabalho envolve muitas atividades repetitivas e de rotina), o efeito marginal do aumento de um desvio-padrão na nota do Inaf eleva em aproximadamente 4 p.p. a probabilidade de um indivíduo estar empregado no comércio. Esse setor caracteriza-se pela maior interação entre os profissionais e os clientes, o que demanda habilidades funcionais que, muitas vezes, não são capturadas por meio das estatísticas usuais de escolaridade (nível e/ou anos de estudo).

Este trabalho busca iniciar um debate sobre a empregabilidade dos trabalhadores brasileiros, em uma época de baixas taxas de desemprego, com especial foco nas habilidades funcionais requeridas no mercado de trabalho. Deste modo, procura ser um primeiro passo em uma discussão que possivelmente pode contribuir para a compreensão atual da dinâmica da produtividade dos trabalhadores brasileiros.

\section{Habilidades, educação e alfabetismo funcional}

Os efeitos das diferentes habilidades adquiridas por trabalhadores, ao longo de suas carreiras, em seus salários e empregabilidade constituem tópico recorrente na literatura de capital humano (BOISSIRE; KNIGHT; SABOT, 1985; BECKER, 1993; MURNANE, 1995; HECKMAN, 1999; CARNEIRO; HECKMAN, 2003). Uma habilidade fundamental para a empregabilidade dos trabalhadores é a capacidade de transformar sua educação formal (anos de estudo ou séries completadas) em uma capacidade funcional. Nos níveis mais básicos, essa capacidade, ou habilidade, é denominada alfabetismo funcional. Ou seja, a capacidade de ler e fazer cálculos matemáticos básicos dentro de um contexto social e de trabalho.

Um exemplo prático desse tipo de habilidade foi estudado por Cathery-Goulart et al. (2009). Os autores avaliaram a habilidade de leitura e compreensão de materiais da área da saúde segundo escolaridade e idade. O método consiste em fornecer materiais, como 
frascos de medicamentos e cartões de agendamento de consultas, para que os indivíduos pesquisados sejam avaliados em suas capacidades de compreensão de texto e conceitos numéricos. Os autores utilizaram testes de correlação parcial e de Pearson e um modelo de regressão múltipla, para verificar a associação entre os scores obtidos pelos indivíduos e sua escolaridade e idade. Cerca de 32\% da amostra mostrou déficits de alfabetização funcional. Entre os idosos ( 65 anos ou mais), essa taxa atingiu 51,6\%. Os autores também encontraram uma correlação positiva entre anos de estudo e scores obtidos. Porém, a correlação entre scores e idade não foi significante, quando os efeitos da escolaridade foram controlados.

O estudo citado é um exemplo importante de como o alfabetismo funcional se relaciona com o nível de escolaridade. Entretanto, certas habilidades funcionais não são precisamente medidas pelo nível de escolaridade e afetam a empregabilidade dos indivíduos. Em geral, os estudos focam na relação entre alfabetismo funcional e salários. A literatura investigando os efeitos do alfabetismo funcional na empregabilidade dos trabalhadores é relativamente esparsa. $^{3}$

Blunch e Verner (2000) analisaram a relação entre alfabetismo funcional e rendimentos dos trabalhadores em Gana. O estudo utilizou o Ghana Linving Standards Survey e o Core Welfare Indicators Questionnaire. Os autores estimaram a regressão de rendimentos em um modelo controlando por seleção por meio do método de mínimos quadrados, em dois estágios (Heckman 2-Step), e controlaram para o nível educacional do indivíduo (anos de estudo), as características individuais e as regionais. Após controlar para a autosseleção usando o estado civil (casado ou solteiro) dos indivíduos, os autores não encontraram uma relação estatisticamente significativa entre o nível de alfabetismo funcional e os rendimentos dos trabalhadores naquele país. Entretanto, no primeiro estágio da regressão, os autores verificaram uma relação positiva e significativa no resultado do teste de alfabetismo funcional aplicado aos pesquisados. Ou seja, os resultados apontam que o nível de alfabetismo funcional não afeta os rendimentos, mas afeta a participação do trabalhador no mercado de trabalho.

Azevedo et al. (2007) investigaram os efeitos do alfabetismo funcional sobre a empregabilidade dos trabalhadores, utilizando dados da Pesquisa Nacional por Amostra de Domicílios (PNAD), da Pesquisa sobre Padrões de Vida (PPV) e da Pesquisa Nacional de Demografia e Saúde (PNDS). As estimativas foram obtidas por meio do método de mínimos quadrados ordinários e os resultados mostram que os efeitos agregados são significativos e positivos. Em particular, o estudo revela que a alfabetização tem um impacto positivo sobre a educação dos filhos e o acesso a postos de trabalho.

Recentemente, Ponczek e Rocha (2011), a partir dos dados da Pesquisa Mensal de Emprego (PME), do IBGE, investigaram as relações entre o rendimento e a empregabilidade dos trabalhadores brasileiros com seus niveis de alfabetismo funcional. Os autores estimaram um modelo de probabilidade linear para calcular os efeitos do alfabetismo funcional

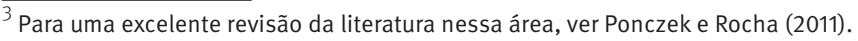


sobre a empregabilidade dos trabalhadores, controlando para características individuais, características regionais, efeito fixo para o mês e ano da pesquisa e escolaridade do pesquisado. O resultado, porém, não se mostrou estatisticamente significativo. Os autores apontam que homens mais velhos, brancos e mais educados possuem maior probabilidade de estarem empregados.

Este artigo busca complementar os trabalhos desenvolvidos recentemente, explorando o indicador Inaf, que ainda não foi utilizado com este propósito, empregando um modelo probit. Enquanto os trabalhos anteriores focavam no efeito do alfabetismo funcional sobre a renda, o presente estudo procura complementar o de Azevedo et al. (2007) e foca na empregabilidade, agregando uma discussão sobre o diferente efeito do alfabetismo funcional sobre as mulheres e de acordo com o setor. Os dados utilizados vão de 2001 até 2007, período similar ao de Ponczek e Rocha (2011), porém neste trabalho é utilizada a pesquisa Inaf, cuja amostra cobre o país inteiro, enquanto a PME contém dados de apenas seis cidades (Recife, Salvador, Belo Horizonte, Rio de Janeiro, São Paulo e Porto Alegre).

\section{Metodologia}

Com o objetivo de entender como o nível de alfabetismo funcional afeta a probabilidade de uma pessoa estar ou não empregada, foi elaborado um modelo de escolha binária, assumindo-se que o termo de erro possui distribuição normal (probit) (GREENE, 2008). A variável dependente é uma dummy, que é igual a 1, se o indivíduo está empregado, e igual a zero, caso contrário. A amostra foi separada entre indivíduos empregados e não empregados (todos os demais). Muitos trabalhos referem-se a estes grupos como ocupados e desocupados. Preferimos não usar esta nomenclatura, pois entre os não empregados encontram-se, por exemplo, mulheres que possuem a ocupação de "dona de casa" e também os estudantes, o que para muitos constitui uma ocupação (apesar de não ser formalmente definida como participação no mercado de trabalho).

O modelo probit consiste em estimar a probabilidade de ocorrer um fato, condicional às características definidas como variáveis independentes, as quais incluem: ano da pesquisa; Estado de residência do entrevistado; características da cidade; características individuais (cor e idade); nível de escolaridade; nível de escolaridade dos pais; e score Inaf do indivíduo. É importante ressaltar uma hipótese que está subjacente à estimação deste modelo. Estamos assumindo que o alfabetismo funcional do indivíduo é uma medida exógena, ou seja, é tomada como dada, quando do momento da decisão da entrada no mercado de trabalho e busca por um emprego. ${ }^{4}$

\footnotetext{
${ }^{4}$ Estamos assumindo que, condicional aos demais fatores observáveis, a correlação entre o score Inaf e o erro de estimativa é zero (exogeneidade). Esta hipótese pode ser questionada se pensarmos que atributos não observáveis (como motivação ou autoestima, por exemplo) afetam tanto a probabilidade de emprego quanto o score Inaf (viés de variável omitida, endogeneidade). Mas este não é um problema fácil de controlar. Mesmo com alguma medida deste tipo de atributo, os resultados podem ser afetados por este tipo de problema. Entretanto, é importante destacar tal aspecto.
} 
As variáveis relativas ao ano da pesquisa buscam captar o fator temporal, ou seja, os efeitos de variações na taxa de emprego da economia como um todo sobre a probabilidade de o entrevistado estar empregado. A variável dummy, que capta as diferenças entre os Estados, procura corrigir as diferenças entre os mercados de trabalho das diferentes regiões do país. As características da cidade foram incluídas para controlar para os efeitos locais sobre a chance de o entrevistado estar empregado. ${ }^{5}$ As características individuais buscam captar as diferenças entre os entrevistados e os padrões dos mercados de trabalho já explorados na literatura, como as diferenças de empregabilidade entre sexos, cores e faixas etárias, por exemplo. Também foram incluídas dummies para capturar o efeito da escolaridade dos pais sobre a empregabilidade dos indivíduos, como forma de conseguir alguma heterogeneidade no nível de habilidade inicial (de origem genética ou de renda) dos indivíduos.

Três especificações do modelo foram estimadas: a primeira contém apenas características individuais; a segunda engloba as características regionais; e a terceira inclui uma interação entre a dummy de sexo e o score Inaf. O objetivo é verificar se o efeito marginal do score Inaf é diferente entre homens e mulheres, uma vez que a literatura de capital humano indica que o acúmulo de habilidades pode se dar de forma diferente entre homens e mulheres. ${ }^{6}$ Para cada uma das três especificações, foram testados dois modelos: um utilizando o score Inaf como variável independente; e outro com dummies que indicam a classificação do indivíduo (analfabeto, rudimentar, básico e pleno), tomando-se como base o nível analfabeto.

Além disso, buscou-se testar a hipótese de que o efeito do ganho de habilidades funcionais é maior entre os indivíduos de menor escolaridade. Para tanto, com o objetivo de não saturar o modelo com diversas interações entre dummies de escolaridade e resultado do score Inaf, optou-se por reduzir a amostra para apenas aqueles indivíduos que possuem escolaridade menor ou igual ao ensino fundamental completo.

Em uma investigação final, buscou-se mensurar o efeito do alfabetismo funcional em três setores distintos: prestação de serviços; indústria de transformação; e comércio. O objetivo é investigar se esses setores requerem diferentes níveis de alfabetismo funcional e se os efeitos sobre a empregabilidade são distintos para homens e mulheres nesses setores, já que o primeiro possui mais mulheres empregadas, o segundo é majoritariamente formado por homens e o terceiro apresenta um equilíbrio entre ocupações femininas e masculinas.

\footnotetext{
${ }^{5}$ Deve-se ressaltar que não foi utilizada uma dummy para cada cidade, pois em muitas cidades a quantidade de indivíduos entrevistados é muito pequena, relativamente.

${ }^{6}$ A literatura econômica de capital humano enfatiza principalmente a distinção na acumulação de habilidades cognitivas e não cognitivas entre homens e mulheres (MURNANE, 1995; HECKMAN, 1999; CARNEIRO; HECKMAN, 2003, CUNHA; HECKMAN; SCHENNACH, 2010). Portanto, parece razoável permitir que a especificação possibilita capturar efeitos diferentes do resultado do score/classificação Inaf entre homens e mulheres.
} 


\section{Descrição da base de dados}

\section{Indicador de Alfabetismo Funcional (Inaf)}

A definição de amostras, a coleta de dados e seu processamento foram feitos pelo Ibope, em apoio à ação social realizada pelo Instituto Paulo Montenegro. Criado em 2001, o Inaf investiga a capacidade de leitura, escrita e cálculo da população brasileira adulta. Entre 2001 e 2005, o Inaf foi divulgado anualmente, alternando as habilidades pesquisadas. Assim, em 2001, 2003 e 2005, foram medidas as habilidades de leitura e escrita (letramento); e, em 2002 e 2004, as habilidades de matemática (numeramento). A partir de 2007, a pesquisa passou a ser bienal, trazendo, simultaneamente, as habilidades de letramento e numeramento e mantendo a análise da evolução dos índices a cada dois anos. ${ }^{7}$

Entretanto, para poder analisar o alfabetismo funcional ao longo do tempo, o Instituto Paulo Montenegro buscou um método para tornar possível essa comparação. Então, foi criado o Indicador Geral do Alfabetismo Funcional. O indicador foi elaborado a partir de uma análise das dimensões associadas aos itens de cada um dos testes - língua portuguesa e matemática -, derivando-se uma única dimensão. Para as habilidades medidas no Inaf, não há uma distinção muito expressiva entre os conhecimentos em língua portuguesa e matemática. Logo, foi possível produzir uma única escala de competências que tornasse a medida comparável ao longo do tempo. ${ }^{8}$

O indicador mensura os níveis de alfabetismo funcional da população brasileira de 15 a 64 anos de idade, englobando residentes em zonas urbanas e rurais de todas as regiões do Brasil, quer estejam estudando ou não. Em entrevistas domiciliares, são aplicados questionários e testes práticos. O intervalo de confiança estimado é de 95\% e a margem de erro máxima estimada é de 2,2 pontos percentuais para mais ou para menos, sobre os resultados encontrados no total da amostra. ${ }^{9}$

A definição de analfabetismo vem, ao longo das últimas décadas, sofrendo revisões significativas, como reflexo das próprias mudanças sociais. Em 1958, a Unesco definia como alfabetizada uma pessoa capaz de ler e escrever um enunciado simples; algo relacionado à sua vida diária. Vinte anos depois, a Unesco sugeriu a adoção dos conceitos de analfabetismo e alfabetismo funcional (INSTITUTO PAULO MONTENEGRO, 2007). Portanto, é considerada alfabetizada funcionalmente "A pessoa capaz de utilizar a leitura, a escrita e as habilidades matemáticas para fazer frente às demandas de seu contexto social e utilizá-las para continuar aprendendo e se desenvolvendo ao longo da vida" (INSTITUTO PAULO MONENEGRO, 2007, p. 1).

\footnotetext{
${ }^{7}$ Os resultados do ano de 2009 não haviam sido divulgados quando do término deste artigo.

${ }^{8}$ De acordo com o instituto responsável pela pesquisa, os itens foram calibrados, reunindo-se todas as bases de dados (de todos os anos), e os modelos foram construídos para todos os itens, considerando-se que eles estariam associados a um único fator latente (o alfabetismo funcional do indivíduo). Simultaneamente, foram produzidas as medidas dos indivíduos expressas nessa escala. Um teste com itens de língua portuguesa e matemática e itens comuns às avaliações anteriores serviu para tornar comparáveis as medidas das diferentes provas.

${ }^{9}$ Para mais detalhes sobre seleção da amostra e estimativas da pesquisa, ver Instituto Paulo Montenegro (2006).
} 
De acordo com a metodologia da pesquisa (INSTITUTO PAULO MONTENEGRO, 2006, 2007), os níveis de alfabetismo funcional são divididos em quatro:

- analfabeto - corresponde à condição dos que não conseguem realizar tarefas simples, que envolvem a leitura de palavras e frases, ainda que uma parcela destes consiga ler números familiares (número de telefone, preços, etc.);

- rudimentar - compreende a capacidade de localizar uma informação explícita em textos curtos e familiares (como um anúncio ou pequena carta), ler e escrever números usuais e realizar operações simples, como manusear dinheiro para o pagamento de pequenas quantias ou fazer medidas de comprimento usando a fita métrica;

- básico - as pessoas classificadas nesse nível podem ser consideradas funcionalmente alfabetizadas, pois já leem e compreendem textos de média extensão, localizam informações, mesmo que seja necessário realizar pequenas inferências, leem números na casa dos milhões, resolvem problemas envolvendo uma sequência simples de operação e têm noção de proporcionalidade. No entanto, essas pessoas mostram limitações quando as operações requeridas envolvem maior número de elementos, etapas ou relações;

- pleno - classificadas nesse nível estão as pessoas cujas habilidades não mais impõem restrições para compreender e interpretar textos em situações usuais: leem textos mais longos, analisando e relacionando suas partes; comparam e avaliam informações; distinguem fato de opinião; realizam inferências e sínteses. Quanto à matemática, elas resolvem problemas que exigem maior planejamento e controle, envolvendo percentuais, proporções e cálculo de área, além de interpretar tabelas de dupla entrada, mapas e gráficos.

O score Inaf é calculado com base em uma escala, criada pelo Instituto Paulo Montenegro (2007), que utilizou as respostas dos testes aplicados e a Teoria da Resposta ao Item (TRI) como metodologia estatística para que fossem analisadas as notas ao longo do tempo. A TRI consiste em modelos teóricos que representam o comportamento da resposta atribuída a cada uma das questões, como uma função habilidade do indivíduo. Calculado dessa forma, o score Inaf resume as habilidades funcionais do indivíduo e permite a comparação entre indivíduos de amostras de períodos diferentes. ${ }^{10}$

\section{Variáveis individuais e regionais}

Além das medidas de proficiência (score Inaf e categoria), a base de dados também fornece características geográficas e socioeconômicas dos indivíduos. As características regionais buscam captar os efeitos das variações conjunturais e macroeconômicas de cada mercado de trabalho, uma vez que a empregabilidade não depende apenas dos aspectos individuais. Já a introdução das características socioeconômicas visa captar as diferenças de empregabilidade entre sexos, cores e faixas etárias, assim como alguma heterogeneidade no nível de habilidade inicial (de origem genética ou de renda) dos indivíduos. Neste estudo, foram utilizados os seguintes conjuntos de variáveis:

\footnotetext{
${ }^{10}$ A metodologia de Teoria de Resposta ao Item (TRI) permite comparar testes que possuem questões (itens) diferentes. Os itens são então calibrados (ponderados) pelos seus níveis de dificuldade e colocados em uma mesma escala. Uma das principais características da TRI é que ela torna possível comparar resultados de provas ou testes diferentes, inclusive ao longo do tempo, como é o caso do teste do Inaf. Para uma descrição mais detalhada da TRI, ver Hambleton, Swaminathan e Rogers (1991).
} 
- ano - ano de realização do teste de proficiência. Para cada ano, foi criada uma variável dummy com o ano base definido como o de 2007;

- Estado - unidade da federação onde o indivíduo se encontrava no momento da realização do teste. Para cada Estado, foi criada uma variável dummy, que é igual a 1, se o indivíduo se encontrava naquele Estado. O Espírito Santo foi utilizado como Estado base;

- cidade - contém características da cidade onde o entrevistado residia no ano da pesquisa. Foram criadas dummies para cada situação da cidade (capital, interior ou periferia), utilizando como base a capital. Foi considerado também o Índice de Desenvolvimento Humano (IDH), variável contínua, no intervalo de 0 a 1. Adicionalmente, foram criadas variáveis dummies para o tamanho da população, em cada faixa definida na pesquisa (até 20 mil habitantes, 20 mil a 100 mil habitantes e mais de 100 mil habitantes), e foi usada como base a categoria "mais de 100 mil habitantes". Finalmente, foi criada uma dummy de localização da cidade (rural e urbana) e usada como base a urbana. O objetivo é caracterizar os diferentes mercados de trabalho locais e demandas por habilidades;

- características individuais - foram utilizadas as seguintes características: idade discreta, no intervalo de 15 a 64 anos; sexo, sendo criada uma dummy (masculino e feminino), tendo como base o sexo masculino; e cor (branca, parda, preta, amarela, vermelha e outra), sendo criadas variáveis dummies para cada cor e usada como base a cor branca;

- escolaridade - para identificar a escolaridade do indivíduo entrevistado, foram estabelecidas dez categorias (analfabeto, sabe ler, mas não frequentou a escola, primário incompleto, primário completo, ginásio incompleto, ginásio completo, colegial incompleto, colegial completo, superior incompleto e superior completo). Foram criadas variáveis dummies para cada categoria e usada como base a categoria superior completo;

- escolaridade dos pais - para identificar as habilidades hereditárias ou nível de alfabetismo funcional durante a infância, foi usada a escolaridade do pai e da mãe. Cada uma tem oito categorias (nenhuma, até a 4⿳亠口冖 série, fundamental incompleto, fundamental completo, médio incompleto, médio completo, superior incompleto e superior completo). Em ambos os casos, foram criadas dummies usando como base o nível superior completo.

\section{Estatísticas descritivas}

A amostra engloba 12.006 pessoas, das quais 6.162 (51,3\%) são do sexo feminino e 5.844 (48,7\%) do masculino. A porcentagem de homens e mulheres em cada nível de classificação de alfabetismo funcional se mantém praticamente em $50 \%$ para cada sexo. Nota-se também que apenas $8 \%$ dos entrevistados pela pesquisa, em todos os anos, são considerados analfabetos funcionais. As diferenças entre homens e mulheres também apontam para uma semelhança estatística entre os dois grupos: em média, 36,97\% das mulheres e 37,28\% dos homens são classificados como analfabetos ou em nível rudimentar.

A amostra é constituída, principalmente, de indivíduos que vivem no meio urbano (84,2\%). Os dados mostram que, na área rural, existe uma maior concentração de indivíduos classificados nos níveis analfabeto e rudimentar. Do total da amostra, entre aqueles que estão no nível analfabeto, 66,8\% encontram-se no setor urbano e 33,2\% no rural, para os de nível rudimentar, 79,5\% estão no urbano e 20,5\% no rural, para os de nível básico, essas proporções correspondem a $86 \%$ e 14\%, respectivamente, e, para os de nível pleno, 92,1\% pertencem ao urbano e $7,9 \%$ ao rural. 
A Tabela 1 apresenta a distribuição dos indivíduos entrevistados, segundo nível de escolaridade e sexo. Os dados mostram a tendência de aumento da escolaridade em ambos os sexos. Em 2001, 46,5\% dos homens possuíam, no máximo, o primário completo, enquanto 41,5\% das mulheres estavam nessa situação. Já em 2007, apenas 33\% das mulheres e $35,7 \%$ dos homens estavam classificados nesse grupo. O nível de escolaridade é uma variável importante neste estudo, pois se deseja investigar a relação entre o nível de alfabetismo, medido pelo Inaf, e a probabilidade de estar empregado, uma vez controlada pelo nível de escolaridade.

É importante ressaltar que a escolaridade e o desempenho no exame de alfabetismo funcional são positivamente correlacionados. Indivíduos com maior escolaridade obtiveram maiores notas, medidas pelo score Inaf. O Gráfico 1 mostra os histogramas da variável score Inaf para quatro faixas de escolaridade selecionadas.

TABELA 1

Distribuição dos indivíduos entrevistados, segundo sexo e escolaridade

Brasil - 2001-2007

\begin{tabular}{|c|c|c|c|c|c|c|}
\hline \multirow{2}{*}{ Sexo e escolaridade } & & & \multicolumn{3}{|c|}{ Em porcentager } \\
\hline & 2001 & 2002 & 2003 & 2004 & 2005 & 2007 \\
\hline \multicolumn{7}{|l|}{ Homens } \\
\hline Analfabeto & 6,8 & 2,6 & 3,5 & 2,0 & 1,3 & 8,4 \\
\hline Sabe ler & 1,6 & 0,5 & 2,0 & 0,5 & 0,6 & 0,0 \\
\hline Primário incompleto & 16,8 & 19,6 & 18,2 & 16,4 & 17,2 & 12,8 \\
\hline Primário completo & 21,2 & 23,6 & 22,3 & 20,4 & 20,3 & 14,5 \\
\hline Ginásio incompleto & 16,1 & 15,6 & 15,7 & 16,7 & 15,6 & 13,6 \\
\hline Ginásio completo & 11,6 & 12,3 & 12,7 & 10,6 & 11,7 & 12,6 \\
\hline Colegial incompleto & 7,1 & 8,5 & 7,1 & 8,0 & 7,9 & 8,5 \\
\hline Colegial completo & 11,7 & 10,3 & 11,3 & 16,7 & 16,6 & 20,5 \\
\hline Superior incompleto & 4,0 & 3,6 & 3,4 & 5,0 & 4,3 & 4,6 \\
\hline Superior completo & 3,1 & 3,5 & 3,8 & 3,7 & 4,3 & 4,4 \\
\hline \multicolumn{7}{|l|}{ Mulheres } \\
\hline Analfabeta & 4,7 & 2,0 & 2,8 & 1,3 & 1,5 & 6,9 \\
\hline Sabe ler & 0,4 & 0,6 & 0,9 & 0,9 & 0,7 & 0,0 \\
\hline Primário incompleto & 14,5 & 16,5 & 16,2 & 14,7 & 15,3 & 12,7 \\
\hline Primário completo & 22,0 & 22,9 & 21,7 & 20,1 & 19,5 & 13,4 \\
\hline Ginásio incompleto & 17,1 & 16,5 & 16,2 & 15,2 & 14,0 & 14,1 \\
\hline Ginásio completo & 11,2 & 11,6 & 12,6 & 11,1 & 11,6 & 10,1 \\
\hline Colegial incompleto & 8,5 & 8,0 & 8,7 & 10,2 & 8,9 & 9,9 \\
\hline Colegial completo & 13,4 & 13,7 & 13,0 & 17,2 & 18,3 & 21,1 \\
\hline Superior incompleto & 4,0 & 4,4 & 4,1 & 4,7 & 4,7 & 5,0 \\
\hline Superior completo & 4,3 & 3,8 & 3,9 & 4,6 & 5,5 & 6,8 \\
\hline
\end{tabular}

Fonte: Instituto Paulo Montenegro 2001-2007.

De acordo com os histogramas, observa-se que a distribuição de resultados torna-se mais semelhante a uma distribuição normal e possui uma média mais elevada, à medida que o nível de escolaridade aumenta. Para o total da amostra, o score Inaf possui média 101,6, 
no período estudado. Porém, para os indivíduos classificados como analfabetos, o score Inaf médio é de 41,1 e, para os com nível superior completo, chega a 136,6. Desse modo, ao ser analisado o efeito do alfabetismo funcional sobre a empregabilidade, deve-se levar em conta o nível educacional. É possível que os ganhos de habilidades funcionais sejam importantes para a empregabilidade apenas para os níveis mais baixos de escolaridade.
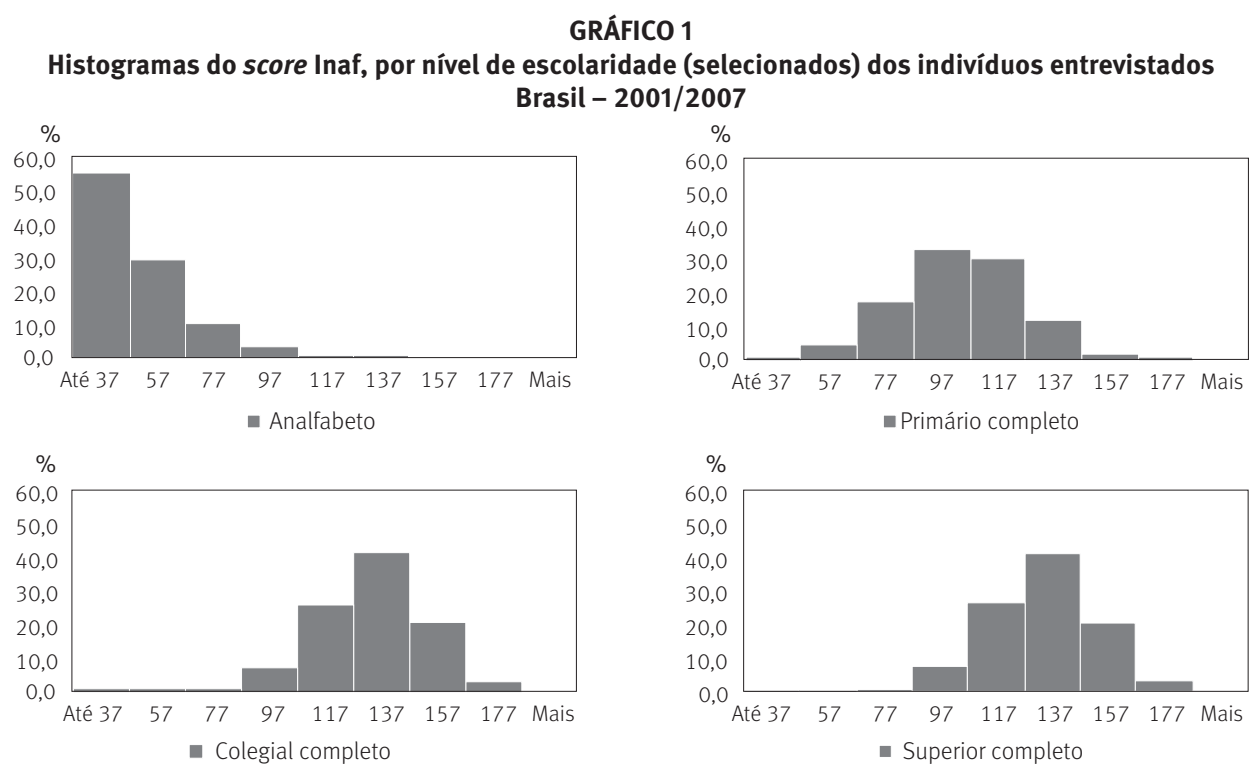

Fonte: Instituto Paulo Montenegro, 2001-2007.

Entre as características individuais, foram utilizadas também a idade e a cor do indivíduo. Os mais jovens apresentaram menor nível de alfabetismo funcional. Na faixa de 15 a 20 anos, $21 \%$ das pessoas estavam nas classificações analfabeto ou rudimentar. Já para aquelas com idade entre 31 e 40 anos, essa porcentagem correspondeu a 37,6\%, evidenciando as diferenças de habilidades funcionais entre as gerações que compõem a amostra.

Os dados apontam que os brancos e amarelos têm uma classificação do Inaf mais alta do que os indivíduos de outras cores. Entre os primeiros, menos de $7 \%$ dos indivíduos eram analfabetos, enquanto para os indígenas e os que se declaram pretos/negros esse percentual superava $10 \%$. Tal resultado aponta para uma heterogeneidade de alfabetismo funcional entre os indivíduos de cores diferentes. Essa estatística é importante, pois pode se refletir no mercado de trabalho e, portanto, na probabilidade de o indivíduo estar empregado.

Analisando os dados de empregabilidade dos pesquisados, nota-se que, em média, 78,6\% dos homens e 52,4\% das mulheres encontravam-se empregados. Destaca-se a relação negativa entre a classificação do Inaf e a proporção de empregados entre os homens nos primeiros anos da pesquisa. Em 2001, enquanto 86,2\% dos homens analfabetos estavam empregados, apenas $72,8 \%$ dos homens com nível de alfabetismo funcional considerado pleno encontravam-se nessa situação. Essa relação se inverte com o passar do tempo e, em 2007, a taxa de emprego era de $77,6 \%$ entre os analfabetos e de $80,7 \%$ entre os de nível pleno. 
Já para as mulheres, a relação positiva entre classificação Inaf e empregabilidade acontece ao longo de todos os anos. Em 2007, por exemplo, 67,59\% das mulheres com nível pleno de alfabetismo estavam empregadas, enquanto apenas 43,01\% das consideradas analfabetas possuíam emprego.

No total da amostra, a proporção de empregados mostrou-se crescente em relação à classificação Inaf: quanto maior a classificação, maior é a proporção de empregados, principalmente nos últimos quatro anos da pesquisa. Observam-se duas tendências importantes. A primeira é o aumento da relação entre classificação Inaf e empregabilidade ao longo do tempo, que pode estar refletindo uma elevação na oferta de empregos que demandam habilidades funcionais. Esse fenômeno pode estar relacionado com o crescimento da participação do setor de serviços na economia brasileira nos últimos anos, vis-à-vis o setor industrial.

Além disso, esse fenômeno é mais acentuado para as mulheres. Em 2001, a diferença entre a porcentagem de mulheres empregadas que possuíam nível pleno e daquelas com nível rudimentar de alfabetismo funcional era 12,3\%. Já em 2007, essa diferença correspondia a $22,2 \%$. Se, de fato, a economia brasileira está passando por uma mudança produtiva do setor industrial para o setor de serviços, o aumento no emprego deve se refletir mais entre as mulheres, já que elas são maioria no setor de serviços (59\% do total) e atividades sociais $(69,5 \%)$.

Portanto, a análise sugere a necessidade de se estudar também o impacto das habilidades funcionais sobre a empregabilidade, permitindo efeitos diferentes para homens e mulheres, bem como entre setores distintos.

\section{Resultados}

A primeira especificação contém apenas características individuais (coluna 1), a segunda inclui as características regionais (coluna 2) e a terceira engloba uma interação entre a dummy de sexo e o score Inaf (coluna 3). Os resultados apresentados na Tabela 2 mostram que o score Inaf tem um impacto positivo sobre a empregabilidade mesmo após ter-se controlado para diversas características individuais, incluindo-se o nível de escolaridade do indivíduo e as características dos mercados de trabalho locais (colunas 1, 2 e 3, respectivamente).

Em média, o efeito marginal ${ }^{11}$ do aumento de um desvio-padrão (aproximadamente 30 pontos) no Score Inaf eleva em 2 p.p. a probabilidade de se estar empregado. Os resultados também indicam que o impacto de uma melhor pontuação obtida no score Inaf é maior (e significativo) entre as mulheres, uma vez controladas as demais características. Em particular, o efeito marginal do aumento de um desvio-padrão no score Inaf eleva em aproximadamente 6\% a probabilidade de a mulher estar empregada. Já entre os homens, o efeito é pouco significativo

\footnotetext{
${ }^{11}$ As tabelas com os resultados dos efeitos marginais não foram reportadas por questão de espaço, mas estão disponíveis e podem ser solicitadas diretamente aos autores. A Tabela 2 e as demais a seguir apresentam os coeficientes do modelo.
} 
estatisticamente e atua, inclusive, na direção contrária. O aumento de um desvio-padrão no score Inaf reduz em 1,5 p.p. a probabilidade de estar empregado.

TABELA 2

Resultados estimados do modelo probit usando o score Inaf (amostra completa) Brasil - 2001/2007

\begin{tabular}{lccc}
\hline \multirow{2}{*}{ Variáveis independentes } & \multicolumn{2}{c}{ Variável dependente: empregado } \\
\cline { 2 - 3 } & Modelo 1 & Modelo 2 & Modelo 3 \\
\hline Score Inaf & $0,0017^{\star \star \star}$ & $0,0016^{\star \star \star}$ & $-0,0014^{\star}$ \\
Sexo feminino & $(0,0005)$ & $(0,0005)$ & $(0,0007)$ \\
& $-0,8389^{\star \star \star}$ & $-0,8380^{\star \star \star}$ & $-1,3949^{\star \star \star}$ \\
Feminino*score Inaf & $(0,0257)$ & $(0,0257)$ & $(0,0884)$ \\
& & & $0,0055^{\star \star \star}$ \\
Características individuais & & & $(0,0008)$ \\
Características regionais & Sim & Sim & Sim \\
\hline Log-verossimilhança & & $-6.651,59$ & Sim \\
Wald & $-6.708,58$ & $1.967,34$ & $-6.628,78$ \\
Pseudo R & $1.849,47$ & 0,1425 & $1.999,62$ \\
\hline
\end{tabular}

Fonte: Instituto Paulo Montenegro, 2001-2007.

Nota:**, ${ }^{\star \star}$ e ${ }^{\star}$ significante a 1\%, 5\% e 10\%, respectivamente. Desvios-padrão robustos entre parênteses. Todas as especificações incluem uma constante e dummies para o ano da pesquisa. Características individuais incluem: cor, idade, idade ao quadrado, escolaridade e escolaridade dos pais. Características regionais incluem: estado, situação da cidade, IDH da cidade, tamanho da população e localização da cidade. $\mathrm{N}=12.006$.

Esse resultado para os homens é contraintuitivo. Uma possibilidade é que o efeito do score Inaf sobre a empregabilidade seja não linear. Para verificar essa hipótese, o mesmo modelo foi estimado, mas a partir de variáveis independentes dummies que indicam a classificação de cada indivíduo na prova Inaf. Os resultados estão apresentados na Tabela 3.

Os novos resultados mostram que, uma vez controladas as diferenças individuais e regionais e permitindo-se que os efeitos do score Inaf sejam diferentes entre homens e mulheres, o impacto da classificação no Inaf é significativo somente para as mulheres. Em particular, uma mulher classificada no nível básico possui uma probabilidade de estar empregada que é 6,6 p. p. maior do que a daquela que está no nível analfabeto. Já uma mulher classificada no nível pleno de alfabetismo funcional se encontra empregada com uma probabilidade que é, aproximadamente, 16,5 p.p. maior do que a daquela no nível analfabeto da escala Inaf.

Nos dois modelos estimados e nas três especificações diferentes, a dummy de sexo foi negativa e significativa a 1\%, capturando a menor taxa de empregabilidade entre as mulheres, relativamente aos homens. Cabe ressaltar que uma parcela dos respondentes que não estão empregados pode estar inativa (fora do mercado de trabalho). Este nível de inatividade é maior entre as mulheres. Neste caso, o coeficiente reflete também esta diferença entre os sexos.

Além disso, as variáveis relacionadas a nível educacional, cor, idade e características da cidade também se mostraram significativas em todas as especificações, ressaltando a importância das características individuais e regionais no mercado de trabalho. Os 
resultados dos efeitos diretos dessas outras variáveis independentes foram omitidos, pois estas servem apenas como controle na medição do efeito direto do Inaf, que é o objetivo principal do artigo.

TABELA 3

Resultados estimados do modelo probit usando a classificação Inaf (amostra completa) Brasil - 2001/2007

\begin{tabular}{|c|c|c|c|}
\hline \multirow{2}{*}{ Variáveis independentes } & \multicolumn{3}{|c|}{ Variável dependente: empregado } \\
\hline & Modelo 1 & Modelo 2 & Modelo 3 \\
\hline \multirow[t]{2}{*}{ Classif. Inaf rudimentar } & 0,0719 & 0,0796 & 0,0380 \\
\hline & $(0,0581)$ & $(0,0587)$ & $(0,0852)$ \\
\hline \multirow[t]{2}{*}{ Classif. Inaf básico } & $0,1405^{\star \star}$ & $0,1550^{\star \star}$ & 0,0466 \\
\hline & $(0,0625)$ & $(0,0630)$ & $(0,8688)$ \\
\hline \multirow[t]{2}{*}{ Classif. Inaf pleno } & $0,1920^{\star \star \star}$ & $0,1819^{\star \star \star}$ & $-0,1108$ \\
\hline & $(0,0685)$ & $(0,0690)$ & $(0,0922)$ \\
\hline \multirow[t]{2}{*}{ Sexo feminino } & $0,8395^{\star \star \star}$ & $-0,8387^{\star \star \star}$ & $-1,0593^{\star \star \star}$ \\
\hline & $(0,0257)$ & $(0,0257)$ & $(0,0915)$ \\
\hline \multirow[t]{2}{*}{ Feminino*classif. Inaf rudimentar } & & & 0,0735 \\
\hline & & & $(0,1030)$ \\
\hline \multirow[t]{2}{*}{ Feminino*classif. Inaf básico } & & & $0,1906^{*}$ \\
\hline & & & $(0,1001)$ \\
\hline \multirow[t]{2}{*}{ Feminino*classif. Inaf pleno } & & & $0,5154^{\star \star \star}$ \\
\hline & & & $(0,1044)$ \\
\hline Características individuais & & Sim & Sim \\
\hline Características regionais & & Sim & Sim \\
\hline Log-verossimilhança & $-6.707,92$ & $-6.650,76$ & $-6.626,54$ \\
\hline Wald & $1.852,45$ & $1.970,81$ & $2.003,46$ \\
\hline Pseudo $\mathrm{R}^{2}$ & 0,1352 & 0,1426 & 0,1457 \\
\hline
\end{tabular}

Fonte: Instituto Paulo Montenegro, 2001-2007.

Nota: ${ }^{* \star *},{ }^{* *} e^{*}$, significante a 1\%,5\% e 10\%, respectivamente. Desvios-padrão robustos entre parênteses. Todas as especificações incluem uma constante e dummies para o ano da pesquisa. Características individuais incluem: cor, idade, idade ao quadrado, escolaridade e escolaridade dos pais. Características regionais incluem: estado, situação da cidade, IDH da cidade, tamanho da população e localização da cidade. $\mathrm{N}=12.006$.

A Tabela 4 apresenta os resultados do modelo quando se restringiu a amostra apenas aos indivíduos com escolaridade igual ou inferior ao ginásio completo (equivalente ao atual ensino fundamental). O objetivo é verificar se o efeito do score Inaf sobre a empregabilidade dos indivíduos é maior para aqueles que possuem menor nível de escolaridade, os quais representam cerca de dois terços da amostra.

Os resultados mostram que, uma vez controladas as características individuais e regionais, um aumento de um desvio-padrão no score Inaf eleva em aproximadamente 4 p.p. a probabilidade de uma mulher que possui nível de escolaridade menor ou igual ao ginásio completo estar empregada. Esse resultado é de magnitude similar, porém um pouco menor do que o encontrado na Tabela 2. Portanto, os efeitos das habilidades funcionais não parecem ser maiores entre aqueles de escolaridade inferior, como se imaginava inicialmente. Esse resultado é relevante, pois mostra que as habilidades funcionais podem ser importantes no mercado de trabalho mesmo para os indivíduos que possuem um nível de escolaridade elevado. 
TABELA 4

Resultados estimados do modelo probit usando o score Inaf (amostra apenas com indivíduos com escolaridade até ginásio completo) Brasil - 2001/2007

\begin{tabular}{|c|c|c|c|}
\hline \multirow{2}{*}{ Variáveis independentes } & \multicolumn{3}{|c|}{ Variável dependente: empregado } \\
\hline & Modelo 1 & Modelo 2 & Modelo 3 \\
\hline \multirow[t]{2}{*}{ Score Inaf } & $0,0017^{\star \star}$ & $0,0019^{\star \star \star}$ & 0,0001 \\
\hline & $(0,0006)$ & $(0,0006)$ & $(0,0008)$ \\
\hline \multirow[t]{2}{*}{ Sexo feminino } & $-0,9497^{\star \star \star}$ & $-0,9502^{\star \star \star}$ & $-1,2517^{\star \star \star}$ \\
\hline & $(0,0313)$ & $(0,0313)$ & $(0,1006)$ \\
\hline \multirow[t]{2}{*}{ Feminino*score Inaf } & & & $0,0033^{\star \star \star}$ \\
\hline & & & $(0,0010)$ \\
\hline Características individuais & Sim & Sim & $\operatorname{Sim}$ \\
\hline Características regionais & & Sim & Sim \\
\hline Log-verossimilhança & $-4.530,59$ & $-4.486,59$ & $-4.481,55$ \\
\hline Wald & $1.350,98$ & $1.433,72$ & $1.436,20$ \\
\hline Pseudo $\mathrm{R}^{2}$ & 0,1446 & 0,1529 & 0,1539 \\
\hline
\end{tabular}

Fonte: Instituto Paulo Montenegro, 2001-2007.

Nota: ${ }^{\star \star \star},{ }^{\star \star} \mathrm{e}^{\star}$, significante a 1\%, 5\% e 10\%, respectivamente. Desvios-padrão robustos entre parênteses. Todas as especificações incluem uma constante e dummies para o ano da pesquisa. Características individuais incluem: cor, idade, idade ao quadrado, escolaridade e escolaridade dos pais. Características regionais incluem: estado, situação da cidade, IDH da cidade, tamanho da população e localização da cidade. $N=8.097$.

Com o objetivo de investigar os efeitos do resultado do score Inaf sobre a empregabilidade de homens e mulheres em diferentes setores, o mesmo modelo foi estimado usando-se três subamostras distintas: prestação de serviços, indústria de transformação e comércio. A Tabela 5 reporta os resultados do modelo estimado para os trabalhadores do setor de prestação de serviços. Os resultados indicam que, uma vez consideradas as características individuais e regionais, o score Inaf não altera a empregabilidade nesse setor. A interpretação direta desse resultado é de que as habilidades funcionais capturadas pelo score Inaf não são significativas após controlar-se por características individuais e regionais. Entretanto, é possível que esse setor demande habilidades que estejam mais relacionadas com a escolaridade formal, uma vez que inclui muitos profissionais liberais.

Desse modo, buscou-se analisar o mesmo modelo usando uma amostra de indivíduos da indústria de transformação, que, na sua maioria, são funcionários de empresas. 0 problema é que esse setor é intensivo em atividades manuais e/ou de rotina. Esperase, portanto, que as habilidades funcionais capturadas pelo score Inaf não sejam determinantes da empregabilidade nesse setor. Os resultados encontram-se na Tabela 6.

Conforme o esperado, o score Inaf não se mostra significativo em nenhuma das três especificações testadas. Em particular, nem mesmo a dummy de sexo feminino ou a interação entre o score Inaf e a dummy de sexo são estatisticamente diferentes de zero. A indústria de transformação, além de ser intensiva em atividades manuais, é majoritariamente formada por homens (66,9\%). 
TABELA 5

Resultados estimados do modelo probit usando o score Inaf (amostra apenas com indivíduos trabalhadores do setor de serviços) Brasil - 2001/2007

\begin{tabular}{lccc}
\hline \multirow{2}{*}{ Variáveis independentes } & \multicolumn{3}{c}{ Variável dependente: empregado } \\
\cline { 2 - 3 } & Modelo 1 & Modelo 2 & Modelo 3 \\
\hline Score Inaf & 0,0016 & 0,0014 & 0,0002 \\
Sexo feminino & $(0,0013)$ & $(0,0013)$ & $(0,0018)$ \\
& $-0,1716^{\star \star \star}$ & $-0,2037^{\star \star \star}$ & $-0,3917^{\star}$ \\
Feminino*score Inaf & $(0,0606)$ & $(0,0613)$ & $(0,2256)$ \\
& & & 0,0018 \\
Características individuais & & & $(0,0020)$ \\
Características regionais & Sim & Sim & Sim \\
\hline Log-verossimilhança & & Sim & Sim \\
Wald & $-1.178,29$ & $-1.158,21$ & $-157,86$ \\
Pseudo R ${ }^{2}$ & 151,49 & 194,69 & 195,67 \\
\hline
\end{tabular}

Fonte: Instituto Paulo Montenegro, 2001-2007.

Nota: ${ }^{* * *},{ }^{* *}$ e *, significante a 1\%, 5\% e 10\%, respectivamente. Desvios-padrão robustos entre parênteses. Todas as especificações incluem uma constante e dummies para o ano da pesquisa. Características individuais incluem: cor, idade, idade ao quadrado, escolaridade e escolaridade dos pais. Características regionais incluem: estado, situação da cidade, IDH da cidade, tamanho da população e localização da cidade. $\mathrm{N}=2.739$.

TABELA 6

Resultados estimados do modelo probit usando o score Inaf (amostra apenas com indivíduos trabalhadores da indústria de transformação)

\begin{tabular}{llll}
\hline \multirow{2}{*}{ Variáveis independentes } & \multicolumn{2}{c}{ Variável dependente: empregado } \\
\cline { 2 - 4 } & \multicolumn{1}{c}{ Modelo 1 } & \multicolumn{1}{c}{ Modelo 2 } & Modelo 3 \\
\hline Score Inaf & 0,0010 & 0,0022 & 0,0027 \\
Sexo feminino & $(0,0020)$ & $(0,0020)$ & $(0,0023)$ \\
& $-0,2462^{\star \star \star}$ & $-0,2433^{\star \star \star}$ & $-0,0934$ \\
Feminino*score Inaf & $(0,0927)$ & $(0,09424)$ & $(0,3768)$ \\
& & & $-0,0001$ \\
Características individuais & & & $(0,0033)$ \\
Características regionais & Sim & Sim & Sim \\
\hline Log-verossimilhança & & Sim & Sim \\
Wald & $-569,00$ & $-542,07$ & $-541,99$ \\
Pseudo R & 123,91 & 196,11 & 196,05 \\
\hline
\end{tabular}

Fonte: Instituto Paulo Montenegro, 2001-2007.

Nota: ${ }^{\star \star *},{ }^{* \star} \mathrm{e}^{\star}$, significante a 1\%, 5\% e 10\%, respectivamente. Desvios-padrão robustos entre parênteses. Todas as especificações incluem uma constante e dummies para o ano da pesquisa. Características individuais incluem: cor, idade, idade ao quadrado,

escolaridade e escolaridade dos pais. Características regionais incluem: estado, situação da cidade, IDH da cidade, tamanho da população e localização da cidade. $\mathrm{N}=1.227$.

Uma análise do impacto das habilidades funcionais sobre a empregabilidade passa, portanto, pela abordagem de um setor que não tenha tantos profissionais liberais, não seja baseado somente em atividades repetitivas e possua um bom equilíbrio entre homens e mulheres. O setor que contém essas características é o comércio. Desse modo, foram 
estimadas as três especificações restringindo-se a amostra aos 1.371 indivíduos empregados no setor comercial (Tabela 7).

TABELA 7

Resultados estimados do modelo probit usando o score Inaf (amostra apenas com indivíduos trabalhadores do setor de comércio)

Brasil - 2001/2007

\begin{tabular}{lccc}
\hline \multirow{2}{*}{ Variáveis independentes } & \multicolumn{2}{c}{ Variável dependente: empregado } \\
\cline { 2 - 4 } & Modelo 1 & Modelo 2 & Modelo 3 \\
\hline Score Inaf & $0,0051^{\star \star}$ & $0,0041^{\star}$ & $0,0058^{\star \star}$ \\
Sexo feminino & $(0,0022)$ & $(0,0021)$ & $(0,0026)$ \\
& $-0,1467^{\star}$ & $-0,1509$ & 0,3245 \\
Feminino*score Inaf & $(0,0844)$ & $(0,0862)$ & $(0,3852)$ \\
& & & $-0,0042$ \\
Características individuais & & & $(0,0033)$ \\
Características regionais & Sim & Sim & Sim \\
\hline Log-verossimilhança & & Sim & Sim \\
Wald & $-574,12$ & $-561,31$ & $-560,53$ \\
Pseudo R & 105,08 & 124,41 & 126,55 \\
\hline
\end{tabular}

Fonte: Instituto Paulo Montenegro, 2001-2007.

Nota: ${ }^{* \star *},{ }^{* *} \mathrm{e}^{*}$, significante a 1\%,5\% e 10\%, respectivamente. Desvios-padrão robustos entre parênteses. Todas as especificações incluem uma constante e dummies para o ano da pesquisa. Características individuais incluem: cor, idade, idade ao quadrado, escolaridade e escolaridade dos pais. Características regionais incluem: estado, situação da cidade, IDH da cidade, tamanho da população e localização da cidade. $\mathrm{N}=1.371$.

No setor comercial, o score Inafé significativo para todos os trabalhadores, não havendo uma importância maior somente para as mulheres, como foi o resultado com a amostra toda (Tabelas 2 e 3). O efeito marginal do aumento de um desvio-padrão na nota do Inaf, mantendo-se constantes as outras variáveis independentes, eleva em aproximadamente 4 p.p. a probabilidade de um indivíduo estar empregado no setor comercial.

O comércio caracteriza-se pela maior interação entre os profissionais e os clientes, o que demanda habilidades funcionais que, muitas vezes, não são capturadas por meio das estatísticas usuais de escolaridade (nível e/ou anos de estudo). Portanto, este resultado demonstra a importância do alfabetismo funcional entre adultos, ressaltando as diferenças entre os sexos e também entre os diferentes setores da economia.

\section{Considerações finais}

Este trabalho utilizou a pesquisa Indicador Nacional de Alfabetismo Funcional (Inaf) para investigar a relação entre alfabetismo funcional e empregabilidade entre os trabalhadores brasileiros. Estimando um modelo probit e controlando para diversas características observáveis (incluindo o nível educacional dos entrevistados), os resultados apontam para uma importância maior das habilidades funcionais entre as mulheres.

Em média, o efeito marginal do aumento de um desvio-padrão no score Inaf eleva em 6 p.p. a probabilidade de uma mulher estar empregada. Utilizando-se o nível de alfabetismo 
funcional (em vez do score bruto), verificou-se que este resultado permanece significativo. Foi feita também uma análise setorial, observando-se que o efeito marginal do aumento de um desvio-padrão no score Inaf eleva em aproximadamente 4 p.p. a probabilidade de um indivíduo estar empregado no setor comercial, caracterizado pelo maior uso de habilidades funcionais, independentemente de ser do sexo masculino ou feminino.

Este resultado contribui para a literatura, pois aponta que os diferentes setores do mercado de trabalho demandam habilidades específicas para cada área de atuação. Em particular, o score Inaf parece estar capturando um conjunto de habilidades que não são plenamente captadas pelos indicadores tradicionais de conhecimento (anos de escolaridade). Neste caso, o resultado indica que pode ser necessária uma revisão dos conteúdos lecionados nas escolas de ensinos fundamental e médio, por exemplo. Ou ainda, que políticas que buscam aumentar a empregabilidade dos trabalhadores, como o ensino profissionalizante, por exemplo, deveriam estar voltadas para a formação destes tipos de habilidades (mais funcionais e específicas de cada setor).

Uma limitação deste trabalho diz respeito aos fatores microcentrados. Neste estudo, não foram consideradas características específicas dos processos produtivos de cada atividade ou ramo, como, por exemplo, se a atividade é intensiva em capital ou trabalho. Uma futura linha de pesquisa relacionada a esse tema seria uma análise do efeito das habilidades funcionais, medidas pelo score Inaf, sobre os rendimentos dos trabalhadores de diferentes ramos de atividade. Uma segunda sugestão de pesquisa seria o uso de modelos que controlem separadamente os rendimentos de homens e mulheres, para esclarecer a maior significância estatística do score Inaf para mulheres. Por fim, uma outra direção para esta pesquisa seria permitir que os efeitos marginais do score Inaf variassem ao longo do tempo, com o objetivo de capturar mudanças na demanda por habilidades funcionais.

\section{Referências}

AZEVEDO, J.; ULYSSEA, G.; MENDONÇA, R.; FRANCO, S. Avaliação do impacto da alfabetização de adultos sobre o desenvolvimento humano: uma análise com dados secundários. Rio de Janeiro: UFF, 2007.

BECKER, G. Human capital: a theoretical and empirical analysis with special reference to education. $3^{a}$ ed. Chicago: The University of Chicago Press, 1993.

BLUNCH, N.; VERNER, D. Is functional literacy a prerequisite for entering the labor market? An analysis of the determinants of adult literacy and earnings in Ghana. Centre for Labour Market and Social Research, 2000 (Working paper, n. 00-05).

BOISSIERE, M.; KNIGHT, J. B.; SABOT, R. H. Earning, schooling, ability and cognitive skills. The American Economic Review, v. 75, n. 5, 1985.

CARNEIRO, P. M.; HECKMAN, J. Human capital policy. NBER, 2003 (Working paper, n. w9495).

CARTHERY-GOULART, M. et al. Performance of a Brazilian population on the test of functional health literacy in adults. Revista de Saúde Pública, v. 43, n. 2, p. 631-638, 2009.

CUNHA, F.; HECKMAN, J.; SCHENNACH, S. Estimating the technology of cognitive and non cognitive skill formation. NBER, 2010 (Working paper, n. 15664). 
GREENE, W. Econometric analysis. New Jersey: Pearson Prentice Hall, 2009.

HAMBLETON, R.; SWAMINATHAN, H.; ROGERS, H. Fundamentals of item response theory. Newburry Park: Sage Publications, 1991.

HECKMAN, J. Policies to foster human capital. NBER, 1999 (Working paper, n. 7288).

IBGE. Pesquisa Nacional por Amostra de Domicílios -Síntese de Indicadores 2011. Rio de Janeiro, 2011. Disponivel em: 〈http://www.ibge.gov.br〉.

INSTITUTO PAULO MONTENEGRO. Indicador de alfabetismo funcional - $\mathbf{5}$ anos: um balanço dos resultados de 2001 a 2005. São Paulo, 2006.

Indicador de alfabetismo funcional - 0 que é Inaf. São Paulo, 2007. Disponível em: 〈http://www.ipm.org.br〉.

MURNANE, R.; WILLETT, B.; LEVY, F. The growing importance of cognitive skills in wage determination. NBER, 1995 (Working paper, n. w5076).

PONCZEK, V.; ROCHA, M. The effects of adult literacy on earnings and employment. São Paulo: EESP, 2011 (C-Micro working paper, n. 5).

\section{Autores}

Cristiano Machado Costa é Ph. D. em Economia pela University of Pennsylvania. Professor do Programa de Pós-Graduação em Ciências Contábeis da Universidade do Vale do Rio dos Sinos - Unisinos.

José Guilherme Cardoso Correa é bacharel em Economia pela Fundação Instituto Capixaba de Pesquisas em Contabilidade, Economia e Finanças - Fucape.

\section{Endereço de correspondência}

Cristiano Machado Costa

Universidade do Vale do Rio dos Sinos

Secretaria do Programa de Pós-Graduação em Ciências Contábeis

Av. Unisinos, 950 - Prédio 5A, Sala 304

São Leopoldo, RS - CEP 93022-000

\section{Abstract}

The effects of functional literacy on Brazilian workers' employment

This paper uses the survey known as the Functional Literacy Indicator (Indicador de Alfabetismo Funcional, Inaf) to investigate the effects of functional literacy on Brazilian workers' employment levels between 2001 and 2007, emphasizing the differences between genders and among economic sectors. We estimate a series of probit models and control for observable individual characteristics. Results show that the effects of functional illiteracy are higher among women. A difference of one standard deviation on the Inaf exam raises the average woman's probability of being employed by 6.1 p.p. However, proficiency on the Inaf exam does not seem to affect the employment level of Brazilian men. The exception is the commercial sector, where a gain of one standard-deviation on the INAF increases the probability of being employed by 4.p.p. The commercial sector is characterized by the interaction between sellers and clients, which demands functional skills that are usually not captured by regular measures of education (years of schooling).

Keywords: Employment. Literacy. Human Capital. 


\section{Resumen}

Los efectos del alfabetismo funcional sobre la empleabilidad de los trabajadores brasileños

Este artículo utiliza la encuesta Indicador de Alfabetismo Funcional (Inaf), realizada entre 2001 y 2007, para investigar los efectos del alfabetismo funcional sobre la empleabilidad de los trabajadores brasileiros, resaltando las diferencias entre los sexos y sectores económicos. Utilizando modelos probit y controlando las características observables de los individuos, los resultados señalan una mayor importancia de las habilidades funcionales entre las mujeres. El efecto del aumento en una desviación estándar en la nota del Inaf resulta en un crecimiento de 6,10 p.p. en la probabilidad de que la mujer se encuentre empleada. El indicador no se muestra significativo en lo que concierne a la empleabilidad de los hombres brasileños, excepto en el sector comercial, en el que el aumento de una desviación estándar en el score Inaf eleva la probabilidad de que un individuo esté empleado en 4 p.p. El sector comercial se caracteriza por la mayor interacción entre los profesionales y los clientes, demandando habilidades funcionales que muchas veces no son capturadas por medio de las estadísticas usuales de escolaridad (nivel y/o años de estudio).

Palabras-claves: Empleabilidad. Alfabetismo. Capital humano.

Recebido para publicação em 05/09/2012

Aceito para publicação em 09/01/2014 
